\title{
Do young children rehearse? An individual-differences analysis
}

\author{
SUSAN E. GATHERCOLE \\ University of Bristol, Bristol, England \\ and \\ ANNE-MARIE ADAMS and GRAHAM J. HITCH \\ Lancaster University, Lancaster, England
}

\begin{abstract}
The issue of whether young children rehearse in auditory memory tasks was investigated across a series of three studies comparing individual differences in articulation rates and memory spans. Applying the principles of the working-memory model, children with faster rates of speaking should have superior memory spans if they engage in rehearsal. Two of the experiments, with 4 -year-old children, failed to establish any significant association between articulation rate and memory span, although both the memory span and articulation rate procedures were found to be highly reliable in this age group. A third experiment confirmed that, as expected, articulation rates and memory spans were significantly associated with one another in adult subjects. The results indicate that, contrary to recent theories of children's short-term-memory development, 4-year-old children do not engage in subvocal rehearsal during auditory memory span tasks.
\end{abstract}

The developmental course of rehearsal is an important yet unresolved issue. For many years psychologists believed that children do not rehearse until they reach about 7 years of age. There were several lines of evidence to support this position. In particular, there is a marked increase in behavioral indications of rehearsal activity such as whispering and lip movements at around this age (Flavell, Beach, \& Chinsky, 1966). Furthermore, children exhibiting such behaviors obtain better scores on short-term-memory tests than those who do not (Keeney, Canizzo, \& Flavell, 1967). Together, these findings fitted neatly with the then-dominant view that rehearsal represents "inner speech," and that the developmental process of internalization of overt speech is not completed until middle childhood (Vygotsky, 1962).

In the past 10 years or so, there has been a reevaluation of the view that young children do not rehearse, primarily arising from the application of the adult "working-memory" model (Baddeley \& Hitch, 1974) to the study of children's short-term memory. According to the current version of the model (Baddeley, 1986), there is a component of working memory that is specialized for the processing and storage of verbal material-the articulatory (or phonological) loop. The loop itself is composed of a phonological short-term store, which maintains verbal material in a phonological code. Information within the phonological store decays with time, unless it is refreshed by articulatory rehearsal. The rehearsal process

This research project was supported by the Economic and Social Research Council. A.-M. Adams is now at the University of Liverpool, England. Requests for reprints should be addressed to S. E. Gathercole, Department of Psychology, University of Bristol, 8 Woodland Road, Bristol BS8 1TN, England. is conceived as operating in real time, although it does not necessarily involve overt articulation.

This model of verbal short-term memory is considerably more detailed than previous models and has the additional benefit of being accompanied by a suite of experimental techniques that allows researchers to directly investigate the separate components of the articulatory loop. The phenomena known as the word length and articulatory suppression effects are closely linked with the process of subvocal rehearsal. In a seminal paper, Baddeley, Thomson, and Buchanan (1975) showed that adults' recall of word lists is much better if the lists are composed of visually presented words that have a short articulatory duration when spoken (e.g., mumps, stoat, and zinc) than if the words are lengthy (e.g., tuberculosis, hippopotamus, and aluminium). If, however, subjects are instructed to engage in irrelevant articulation during presentation of the memory list (e.g., repeatedly saying "the, the, the'), the recall advantage of short over long words typically disappears.

The explanation for this pattern of findings is that sensitivity to both word length and articulatory suppression are due to rehearsal. The word length effect is suggested to arise because lengthy words take longer to rehearse than short ones, hence increasing the likelihood that memory items held in the phonological store will decay between successive rehearsals. The shorter words will receive more frequent rehearsals and therefore will be better recalled. On the other hand, it is suggested that articulatory suppression prevents subjects from rehearsing. Under suppression conditions, therefore, the benefit of rehearsing short over long words is lost.

The application of the working-memory model and these techniques to the study of verbal short-term-memory 
skills in children led to a surprising conclusion: Children as young as 4 years of age are sensitive to the length of the memory items, providing that the stimuli are presented auditorily (Hitch, Halliday, Dodd, \& Littler, 1989; Hulme, Thomson, Muir, \& Lawrence, 1984). Thus, children, like adults, are better at recalling memory items with one-syllable rather than three-syllable names. The direct implication of this finding is that, contrary to previous beliefs, young children can rehearse and spontaneously choose to do so for auditory speech material. When the memory material is presented in pictorial form, however, sensitivity to word length does not emerge until about 8 years of age (Hitch \& Halliday, 1983).

Although this working-memory perspective on shortterm memory development has been widely accepted, the notion that sensitivity to word length necessarily arises from the use of subvocal rehearsal has been challenged recently. Work on adult short-term memory by Cowan et al. (1992) has established that word length effects can arise simply as a consequence of the delay at recall, resulting from the recall of lengthy rather than short items. This raises the possibility that the sensitivity of young children to the word length of memory items is a consequence of differential output delay in recalling lists of lengthy rather than short items, rather than a result of employing rehearsal. This interpretation receives direct support from the report by Henry (1991a) that 5-year-old children are not impaired by articulatory suppression-a finding also obtained by Gathercole, Willis, and Baddeley (1992). The clear implication of this finding is that, despite the robust findings that young children are sensitive to word length for auditory memory lists (e.g., Hitch et al., 1989; Hulme et al., 1984), they may not rehearse the items. If they do, they should be impaired at recalling memory lists while engaging in articulatory suppression.

Support for the view that young children do not rehearse auditory memory items is also provided in experiments reported by Heffernan, Hitch, and Halliday (1993). As expected, 5-year-old children were found to be better at recalling lists of short rather than long items. These young children were not, however, disrupted by the presentation of memory lists of unknown lengths, in contrast to older children and adults. The effect of unknown list length is typically attributed to its disruptive influence on the use of rehearsal strategies, so this pattern of findings again dissociates young children's sensitivity to the word length effect in the memory span task from other indicators of rehearsal activity.

In the present article, we report a series of experiments that were designed to establish whether children of preschool age do indeed engage in subvocal rehearsal in short-term-memory tasks. These experiments differ markedly from previous work on this issue in that we have adopted an individual-differences rather than an experimental approach. Baddeley et al. (1975) found a high correlation between individual differences in articulation rate and memory scores in adults and took this as further support for the idea that subvocal rehearsal offsets trace decay in the loop component of working memory. The rationale of each of the present experiments is simple: If young children do rehearse in auditory short-termmemory tasks, those with faster rates of explicit articulation should have greater memory spans, because their rapid rehearsal will result in less memory representation loss from the phonological store due to decay. Note that in the present study there is no reliance on the problematic word length index of rehearsal; the focus is simply on whether articulation rate correlates highly with memory span in young children-a correlation that should be found if they use a strategy of subvocal rehearsal.

Using this approach, researchers have shown that children just under 3 years of age do not rehearse in a digit span task; the children's rates of articulation were not significantly correlated with their digit spans (Gathercole \& Adams, 1993). These children were, however, considerably younger than the 4- and 5-year-old subjects from other studies, who were found to be sensitive to word length in recall (e.g., Hitch et al., 1989; Hulme et al., 1984). It is therefore quite plausible that the spontaneous use of rehearsal emerges at some time between 2 and 4 vears of age. In order to test this hypothesis in Experiment 1 , a large sample of 4-year-old children were tested on auditory digit span and speed of articulation for pairs of short and long words. If the children engage in subvocal rehearsal, then memory span and articulation rate measures should be highly associated with one another.

\section{EXPERIMENT 1}

\section{Method \\ Subjects \\ A sample of 71 children participating in an ongoing longitudinal project on the cognitive skills of preschool children were tested for the purposes of this experiment. The children were originally recruited for the longitudinal sample from play groups and via the registry of birth records; see Gathercole and Adams (1993) for a demographic profile of the sample at the first wave of the study. The mean age of the sample was 4 years, 1 month $(S D=.901$ months, range $=3$ years, 11 months to 4 years, 4 months).}

\section{Materials and Procedure}

Testing took place in the child's home in the presence of a caregiver. A battery of language-based tests was administered to the child, including the following measures, which are the focus of this article.

Auditory digit span. On each trial, the experimenter spoke aloud sequences of digits, which the child attempted to repeat immediately in the same sequence. The lists were sampled randomly and without replacement from the sequence 1-9. At least two lists of digits were given at each length, starting at sequence length 2 . If the first two sequences at each length were correctly repeated, the length of the next list was increased by one, and two more lists were presented. If the child failed to repeat both of the two lists at one length, no further lists were given. When the child correctly recalled only one of the first two lists at a particular length, a third list of the same length was given. If the third list was correctly repeated, trials at the next length were given. If the child incorrectly repeated the third list, testing stopped. Span was scored as 
the maximum length at which the child correctly recalled at least two lists. Memory span was scored as " 1 " for any child who failed to correctly recall either of the two-item sequences.

The test-retest reliability of this auditory digit span measure was assessed with forty-five 5-year-old children, with a 4-week interval between successive administrations. The correlation coefficient between test and retest span scores was .66, establishing an acceptable level of reliability.

Articulation rate. Each child's articulation rate was measured on the basis of three repetitions of a pair of one-syllable words (sun and boat) and a pair of three-syllable words (holiday and elephant). The child was given the following instructions: "I would like you to say the two words [sun and boat, or holiday and elephant] one after the other again and again, as quickly as possible, until I tell you to stop." The speed of the repetitions was therefore emphasized, following the conventional procedure for obtaining articulation measures (Hitch \& Halliday, 1983; Hulme et al., 1984). The child was stopped after three full repetitions, and the time taken to complete the three repetitions of each word pair was measured by a stopwatch. From these values, the mean number of words articulated per second was calculated for the one- and three-syllable words for each child.

Nonverbal ability. Each child was tested on the puzzle-solving subtest of the McCarthy Scales of Children's Abilities (McCarthy, 1970 ) in order to provide a measure of their general intellectual skills. This test requires the assembly of a series of six puzzles to form pictures of familiar objects. Successive puzzles increase in complexity, with more points awarded for successful completion of the later puzzles in the series. Maximum score on the test is 27.

\section{Results and Discussion}

The mean digit span score was $2.94(S D=.69$, range $=1-5$ ). The mean articulation rate for the one-syllable words was 3.81 words $/ \mathrm{sec}(S D=.86$, range $=2.34-$ $6.18)$. For the three-syllable items, the mean articulation rate was 1.61 words $/ \mathrm{sec}(S D=.29$, range $=.82-2.59)$. These articulation rates are slightly faster than those obtained from other groups of children of comparable ages (Hitch, Halliday, \& Littler, 1993), indicating that the children participating in the present experiment did indeed treat the articulation task as speeded. The mean score on the nonverbal test was $9.451(S D=4.85$, range $=2-24)$.

The correlations between digit span scores, articulation rates for the one- and three-syllable words, nonverbal test scores, and age are shown in Table 1. The most important feature of the correlation matrix is that digit span was not significantly correlated with either of the articulation rate measures $(r=.19$ for both the one-syllable and threesyllable words). The two articulation rate measures were, however, significantly associated with one another $(r=$

Table 1

Correlations Between Children's Digit Span Scores, Articulation Rates, and Other Measures in Experiment 1

1 Digit span

$\begin{array}{lllll}1 & 2 & 3 & 4 & 5\end{array}$

2 Articulation rate: one-syllable words

3 Articulation rate: three-syllable words

4 Nonverbal ability

1

$19 \quad 1$

$\begin{array}{rr}19 & 1 \\ 19 & 47^{*}\end{array}$

$19 \quad .47 * 1$

$\begin{array}{llll}.40 * & .07 & .03 & 1\end{array}$

5 Age

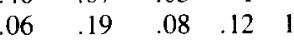

Note-Degrees of freedom are 69 in each case. *Significant at the $1 \%$ level.
$.47, p<.001$ ), establishing a high degree of stability to the rate estimates. The only remaining significant correlation was between digit span and nonverbal ability $(r=$ $.40, p<.001$ ). The finding that digit span scores were highly associated with the children's general intellectual abilities lends credence to the validity of the span measure. Note that the absence of significant correlations between the digit span scores and articulation rate measures in this study does not appear to be due to low statistical power. The power value of the one-tailed significance test for correlations in this study is .96 for a correlation coefficient of $.40,{ }^{1}$ exceeding the conventional desired power value of .8 (Cohen, 1977).

The measures of nonverbal ability and chronological age were included to control for the general intellectual factors that may yield significant correlations between pairs of measures. However, the critical correlations between digit span and the two articulation rate measures failed to reach significance, despite the common positive associations of all three measures with both age and nonverbal scores, so there was no need for further exploration of the specificity of the link between span and articulation rate by using partial correlations.

This pattern of results extends previous findings that speed of articulation and digit span are not reliably associated with one another in 2-year-old (Gathercole \& Adams, 1993) to 4-year-old children. It is not likely that the absence of a significant association between memory span and articulation rate in Experiment 1 was due to a lack of sensitivity or statistical power; the power estimate based on the sample size was very high, and both the digit span task and the articulation measures show acceptable levels of reliability. The simplest interpretation of this finding is that the children of both ages did not use a strategy of subvocal rehearsal to support performance on the digit span measure. If the children did use a rehearsal strategy, we would expect those with the fastest articulation rates to be most effective at maintaining active representations of memory items in the phonological store. Therefore, they should have had larger digit spans.

The interpretation that our 4-year-old children did not rehearse in the span task is clearly at odds with the widely held view that young children do rehearse auditorily presented material in immediate-serial-memory tasks (Hitch \& Halliday, 1983; Hulme et al., 1984). It is therefore important to consider other explanations of the present results that could reconcile our findings with previous research. One possibility is that the comparison between memory span for digits and articulation rates for familiar words is not sufficiently direct. Previous studies that have established highly linear relationships between articulation rate and recall across memory items varying in spoken duration have derived both measures from the same pool of stimuli (Hitch et al., 1989; Hulme et al., 1984; Hulme \& Tordoff, 1989). Note that these studies were not investigations of the linearity of the relationship between rate and memory performance at the level of individual subjects, as in the present study; instead, means of artic- 
ulation rate and recall were plotted across groups of subjects, for each type of materials. The present findings are therefore not directly inconsistent with earlier studies. Nonetheless, before strong claims about the absence of rehearsal can be justified, it is important to rule out the possibility that span and articulation rate in the 4-yearold children participating in the present experiment were unrelated simply because different verbal stimuli were used to make the two kinds of estimate.

Experiment 2 was designed to establish whether memory span and articulation rates would be significantly associated with one another in 4-year-old children when the same verbal materials were used to derive both estimates. Two sets of stimuli were used to yield span and articulation rate measures: digits and familiar concrete nouns. The two pools of stimuli were employed in order to test the generality of any links found between span and rate measures and also as a means of providing a direct assessment of the reliability of each of the two measures with children of this age.

\section{EXPERIMENT 2}

\section{Method}

\section{Subjects}

Forty of the children who had participated in Experiment 1 were tested again, approximately 5 months later, when the mean age of the group was 4 years, 5 months $(S D=1.84$ months, range $=4$ years, 3 months to 4 years, 10 months).

\section{Materials and Procedure}

Each child was tested individually in a single session, which took place in the child's home in the presence of a caregiver. The data were based on the following five measures obtained from each child; the procedure for determining span that was reported in Experiment 1 was used for the two memory span measures.

Digit span. The digits for each memory list were drawn randomly and without replacement from 1-9, omitting the bisyllabic digit 7 .

Word span. Six high-frequency concrete nouns were used in the word span task: balloon, circle, flower, giraffe, puppet, and rabbit. The words were chosen because their estimated ages of acquisition have been shown to be very low, according to the norms reported by Walley and Metsala (1992). The words ranged between 1.29 and 2.47 in their age-of-acquisition ratings, with a mean score of 1.81 ; this value corresponds to an age of acquisition of just under 3 years. Imageability ratings of the words were high, with a mean value of 5.97 on a scale from 1 (low imageability) to 7 (high imageability). Mean standard frequency index was 50.73 (from Kŭcera \& Francis, 1967). The memory lists at each length were constructed by sampling randomly and without replacement from the word pool.

Articulation rates. Two measures of articulation rate were obtained-one for each of the sets of digits and words used to estimate memory span. Articulation rate for each set of stimuli was based on the child's repeating each of three pairs of stimuli from the set three times. For example, word rate estimates were based on three repetitions each of the pairs balloon-giraffe, puppet-circle, and flower-rabbit. As in Experiment 1, the child was instructed to repeat each word pair continuously and as quickly as possible until asked to stop by the experimenter. The child's repetitions were recorded on an audio cassette, and the recording was digitized by using a speech editing package for the Apple Macintosh microcomputer. The time that it took the child to produce the three repetitions was measured directly from the digitized speech waveform. The three separate durations of time that were taken to produce pairs for each set of stimuli were combined to provide a mean articulation rate (in words per second) for each child, for both words and digits.

Nonverbal ability. Raven's Progressive Coloured Matrices test (Raven, 1977) was administered to each child. The test, widely used to provide an estimate of nonverbal intellectual ability in children (Stanovich, Cunningham, \& Feeman, 1984), requires the child, on each trial, to select which of six segments is the missing piece of a visuospatial pattern.

\section{Results and Discussion}

The mean memory span scores were 3.17 for the digits $(S D=.71$, range $=2-5)$ and 3.12 for the words $(S D=$ .52 , range $=2-4)$. The mean articulation rates were 3.519 digits/sec $(S D=.49$, range $=2.80-4.56)$ and 2.17 words $/ \sec (S D=.35$, range $=1.62-2.97)$. On Raven's test of nonverbal ability, the mean raw score was 12.2 $(S D=3.15$, range $=5-18)$. The mean value corresponds to the 50th centile point for children aged 4 years 6 months (Raven, 1984).

The correlations between each of the pairs of measures are shown in Table 2; the findings are straightforward. Digit span and word span scores were highly associated with one another $(r=.57, p<.001)$, as were digit and word articulation rates $(r=.66, p<.001)$. Both the memory span and articulation rates therefore appear to be highly reliable for this age group. However, there was no indication of a positive association between memory spans and articulation rates, despite the use of common stimuli in both procedures. The correlation between digit span and digit articulation rate was .04; the corresponding correlation between word span and word articulation rate was -.09 . The only remaining significant association was between digit span and scores on the nonverbal test $(r=.50, p<.001)$. Note that the power value of the one-tailed significance test in this study was .83 for a correlation coefficient of .4 (Cohen, 1977). Therefore, it is unlikely that the absence of significant correlations between the memory span and articulation rate measures were due to a lack of statistical sensitivity in the study.

Span and articulation rate measures are independent of one another, so further explorations of their relationship to control for differences in age and nonverbal ability were not required. However, it should be noted that after differences due to age and nonverbal ability had been controlled, digit span scores accounted for a further $20.4 \%$ of variance in word span scores $\left[F(1,36)=12.60, M S_{\mathrm{e}}=\right.$

Table 2

Correlations Between Children's Memory Span Scores, Articulation Rates, and Other Measures in Experiment 2

\begin{tabular}{llcrrrr}
\hline & 1 & 2 & 3 & 4 & 5 & 6 \\
\hline 1 Digit span & 1 & & & & & \\
2 Digit articulation rate & .04 & 1 & & & & \\
3 Word span & $.57^{*}$ & -.11 & 1 & & & \\
4 Word articulation rate & .05 & $.66^{*}$ & -.09 & 1 & & \\
5 Nonverbal ability & $.50^{*}$ & .01 & .28 & .01 & 1 & \\
6 Age & .16 & .25 & .18 & .15 & .21 & 1 \\
\hline
\end{tabular}

Note-Degrees of freedom are 38 in each case. *Significant at the $1 \%$ level. 
$.19, p<.005]$. Correspondingly, digit articulation rates were associated with a further $40.8 \%$ of variance in word articulation rates after age and nonverbal ability scores had been taken into account $\left[F(1,36)=25.82, M S_{\mathrm{e}}=\right.$ $.071, p<.001]$. Once again, the strong specific links between these two pairs of measures with different materials attest to their reliability as estimates of short-termmemory ability and articulation speed, respectively.

This clear-cut pattern of findings replicates the results from both the present Experiment 1 and our earlier study with 2-year-old children (Gathercole \& Adams, 1993) and provides strong support for the view that young children do not rehearse in verbal memory span tasks. Memory spans and articulation rates were completely independent of one another for both words and digits. However, the two estimates of memory span were highly associated with one another, as were the two measures of articulation rate. These positive relationships are important, because they establish that both the span and rate measures provided reliable estimates of cognitive skills, even though they failed to share any relationship with one another. The absence of a link between articulation rate and memory span therefore cannot reflect insensitivities of either measure.

Before concluding, on the basis of these results, that young children do not in fact engage in articulatory rehearsal, it seemed important to make a final check of the validity of the individual-differences approach to the issue of whether or not children rehearse. The independence of the children's memory spans and articulation rates was compared with the articulation rate/memory span relationship of a group that is known to rehearse, in order to test the assumption that individual differences in articulation rates influence memory span in rehearsing subjects. If articulation rates and memory span scores are highly correlated with one another in such populations, the independence between the two types of measure in young children can be taken as a genuine indicator that rehearsal is absent. The finding that spans and rates are independent in rehearsing subjects, on the other hand, would invalidate the conclusion that 4-year-old children do not rehearse.

There is, of course, extensive evidence that adults rehearse in immediate-memory tasks (see Baddeley, 1986, for review). In Experiment 3, therefore, we obtained measures of articulation rates and memory spans for a group of adult subjects. As in Experiment 2, subjects were tested for articulation rate and memory span on two sets of stimuli-words and digits. If the findings show that articulation rates and memory spans for each type of stimulus are highly correlated with one another, this would provide a direct endorsement of the interpretation that the absence of such links indicates that a strategy of subvocal rehearsal is not being used. Independence between the measures in this adult subject population would, however, cast serious doubt on the utility of articulation rate and memory span functions as indicators of rehearsal activity.
Table 3

Correlations Between Adults' Memory Spans and Articulation Rates in Experiment 3

\begin{tabular}{lcccc}
\hline & 1 & 2 & 3 & 4 \\
\hline 1 Digit span & 1 & & & \\
2 Digit articulation rate & $.43^{*}$ & 1 & & \\
3 Word span & $.52^{*}$ & $.54^{*}$ & 1 & \\
4 Word articulation rate & $.44^{*}$ & $.79^{*}$ & $.45^{*}$ & 1 \\
\hline
\end{tabular}

Note-Degrees of freedom are 38 in each case. *Significant at the $1 \%$ level.

\section{EXPERIMENT 3}

\section{Method}

\section{Subjects}

Forty undergraduate students from the Department of Psychology at Lancaster University participated in the experiment for pay.

\section{Materials and Procedure}

Each subject provided four measures: memory spans for both digits and words and articulation rates for both digits and words. The procedures for determining span and articulation rates were the same as those described in Experiment 2. It was, however, necessary to expand the two pools of six stimuli in order to yield list lengths of greater than six items. Two new high-frequency, concrete two-syllable words (brother and cartoon) were added to the word pool in order to yield list lengths of up to nine items. Digit lists up to eight items in length were constructed by using the same digit pool employed in Experiment 1.

\section{Results and Discussion}

Mean memory span scores were 6.67 for digits $(S D=$ 1.31 , range $=4-9)$ and 4.82 for words $(S D=.78$, range = 3-6). Mean articulation rates were $5.86 \mathrm{digits} / \mathrm{sec}$ $(S D=.70$, range $=4.45-7.47)$ and 3.66 words $/ \mathrm{sec}$ $(S D=.35$, range $=2.82-4.59)$.

The correlations between the measures are shown in Table 3. All of the measures correlated significantly with one another ( $p<.01$, in each case). Most importantly, digit span and digit articulation rates were highly associated with one another $(r=.43)$, as were span scores and articulation rates for the word stimuli $(r=.45)$.

These findings provide a very clear endorsement of the use of the association between articulation rate and memory span as a means of identifying whether or not subjects have used subvocal articulatory rehearsal in memory span tasks. Positive associations between articulation rate and memory span were found for the adults. In addition, the comparable reliabilities between the pairs of memory span and articulation rate measures across the two age groups in Experiments 2 and 3 rule out any concern that either procedure may be insensitive when applied to young children. The measures appear to be equally robust for both young children and adults.

\section{GENERAL DISCUSSION}

In two experiments, no significant association was found between the articulation rates of 4-year-old children and 
their immediate memory spans. Articulation rate and memory span were, however, closely linked with one another in adults. These results indicate that, contrary to the standard working-memory hypothesis of memory development (Hitch \& Halliday, 1983; Hulme et al., 1984), young children do not use subvocal rehearsal as a means of maintaining auditorily presented material in the articulatory loop component of working memory.

This conclusion fits well with other recent findings that have suggested that young children either do not rehearse at all (Heffernan, Hitch, \& Halliday, 1993; Henry, 1991a, 1991b), or employ a more rudimentary form of rehearsal than the sophisticated cumulative strategy used by older children and adults (Gathercole \& Hitch, 1993; Hitch, 1990; Hitch, Halliday, Schaafstal, \& Heffernan, 1991). The earlier view that young children do rehearse was grounded in demonstrations of their sensitivity to word length in immediate-memory tasks (Hitch et al., 1989; Hulme et al., 1984). It has now become apparent that sensitivity to word length alone is not a sufficient condition to identify rehearsal. In particular, detailed explorations of the mnemonic consequences of varying word length have established that word length effects in adults arise, in part, as a consequence of the inevitable delay to output that is introduced by employing memory items of long spoken durations (Cowan et al., 1992). This view has been reinforced by a recent connectionist simulation of the articulatory loop (Burgess \& Hitch, 1992); the word length effect emerged in the model even when the list rehearsal mechanism was not operational. Once again, the reason was the additional delay, and hence decay, caused by the output of lengthier items. Together, these findings indicate that young children's sensitivity to word length in immediate verbal recall tasks provides an unsubstantial basis for concluding that they do rehearse, in the absence of other converging evidence. Furthermore, it now seems that some elaboration or revision of the workingmemory model (Baddeley, 1986) is required to accommodate the fact that rehearsal is not necessarily involved in the word length effect.

An alternative account of the present results is that young children do in fact rehearse, but that the covert articulatory activity involved is not speeded in the same way as adults' rehearsal. Instead, children may subvocally articulate parts or all of memory sequences in either a more leisurely way, or in a way that is altogether less systematic. This view is certainly consistent with the present findings of a qualitative difference in the memory span of 4year-old children and adults in terms of the sensitivity of memory span to articulatory speed; by this alternative account, though, the qualitative difference is located in the type of rehearsal (speeded or nonspeeded) rather than in its presence or absence. We favor the latter view-that the source of this dissociation lies in the absence of rehearsal in the younger age group-on the grounds of its convergence with other current sources of evidence. However, the opposing view-that young children do rehearse, but in a way that is not sensitive to the articulatory dura- tion of the memory items-remains an interesting and provocative possibility, and one that merits further investigation. Most importantly, though, both accounts directly challenge the notion that rehearsal activity in young children and adults cannot be differentiated.

A question remains: If the young children who participated in Experiments 1 and 2 were not rehearsing the memory stimuli in the span tasks, then how did they manage to maintain the memory sequence? Several of the children from both experiments had memory spans of five items, demonstrating memory skills that are, by any standards, reasonable. According to the working-memory model, the answer to this question is that the spoken memory items gain automatic access to the phonological shortterm-store component of the phonological loop, where they remain until rendered indiscriminable and are finally lost through decay. Without rehearsal, the children could not offset this inevitable process of decay. The present results therefore suggest that some children may have very slow rates of decay in their phonological stores, providing them with functional phonological loop capacities of up to five items, even without the benefits of rehearsal. Although high, this estimate fits reasonably well with demonstrations showing that preventing adults from rehearsing by requiring them to engage in articulatory suppression has disruptive, although by no means disastrous, consequences for recall (Baddeley et al., 1975; Salamé \& Baddeley, 1982). Even without rehearsal, the phonological store itself appears to be sufficient to sustain a significant memory load. In addition, of course, there may be other types of memory representation that can be used to support memory span. In particular, long-term-memory knowledge about the stimuli in the memory sequence is also a significant contributor to immediate-memory performance (Hulme, Maughan, \& Brown, 1991) and may be especially important in young children as a means of supplementing a rehearsal-less phonological loop system.

A more precise account of the development of verbal short-term memory must await further research. The impact of the current results is to constrain such an account by adding further and independent weight to the increasing body of evidence showing that young children, despite having access to the phonological loop, simply do not engage in adult-like subvocal rehearsal.

\section{REFERENCES}

BadDeley, A. D. (1986). Working memory. Oxford: Oxford University Press.

BADDELEY, A. D., \& Hitch, G. J. (1974). Working memory. In G. Bower (Ed.), The psychology of learning and motivation (Vol. 8, pp. 47-90). New York: Academic Press.

BadDeley, A. D., Thomson, N., \& Buchanan, M. (1975). Word length and the structure of short-term memory. Journal of Verbal Leaming \& Verbal Behavior, 14, 575-589.

BuRGESs, N., \& Hitch, G. J. (1992). Toward a network model of the articulatory loop. Journal of Memory \& Language, 31, 429-460.

COHEN, J. (1977). Statistical power analysis for the behavioral sciences (rev. ed.). New York: Academic Press

Cowan, N., Day, L., Saults, S., Keller, T., Johnson, T., \& 
Flores, L. (1992). The role of verbal output time in the effects of word length on immediate memory. Journal of Memory \& Language, 31, 1-17.

Flavell, J. H., Beach, D. R., \& Chinsky, J. M. (1966). Spontaneous verbal rehearsal in a memory task as a function of age. Child Development, 37, 283-299.

Gathercole, S. E., \& Adams, A.-M. (1993). Phonological working memory in very young children. Developmental Psychology, 29. 770-778.

Gathercole, S. E., \& Hitch, G. J. (1993). Developmental changes in short-term memory: A revised working memory perspective. In A. Collins, S. E. Gathercole, M. A. Conway, \& P. E. Morris (Eds.), Theories of memory. Hove: Erlbaum.

Gathercole, S. E., Willis, C. S., \& Baddeley, A. D. (1992). Young children's sensitivity to phonological similarity and articulatory suppression in auditory short-term memory. Unpublished manuscript.

Heffernan, T .M., Hitch, G. J., \& Halliday, M. S. (1993). Dissociation of the word length effect and active rehearsal in young children. Manuscript submitted for publication.

HENRY, L. A. (1991a). Development of auditory memory span: The role of rehearsal. British Journal of Developmental Psychology, 9, 493-511.

Henry, L. A. (1991b). The effects of word length and phonemic similarity in young children's short-term memory. Quarterly Journal of Experimental Psychology, 43A, 35-52.

HiтcH, G. J. (1990). Developmental fractionation of working memory. In G. Vallar \& T. Shallice (Eds.), Neuropsychological impair ments of short-term memory. Cambridge: Cambridge University Press.

Hitch, G. J., \& Halliday, M. S. (1983). Working memory in children. Philosophical Transactions of the Royal Society of London: Series $B, 302,324-340$.

Hitch, G. J., Halliday, M. S., Dodd, A., \& LitTler, J. E. (1989). Development of rehearsal in short-term memory: Differences between pictorial and spoken stimuli. British Journal of Developmental Psychology, 7, 347-362.

Hitch, G. J., Halliday, M. S., \& LitTler, J. E. (1993). Development of memory span for spoken words: The role of rehearsal and item identification processes. British Journal of Developmental Psychology, 11, 159-170.

Hitch, G. J., Halliday, M. S., SchaAfstal, A. M., \& Heffernan, T. M. (1991). Speech, "inner speech," and the development of shortterm memory: Effects of picture-labeling on recall. Journal of Experimental Child Psychology, 51, 220-234.
Hulme, C., Maughan, S., \& Brown, G. D. A. (1991). Memory for familiar and unfamiliar words: Evidence for a long-term memory contribution to short-term memory span. Joumal of Memory \& Language, 30, 241-253.

Hulme, C., Thomson, N., Muir, C., \& Lawrence, A. (1984). Speech rate and the development of short-term memory span. Journal of Experimental Child Psychology, 38, 241-253.

Hulme, C., \& TORDOFF, V. (1989). Working memory development: The effects of speech rate, word length, and acoustic similarity on serial recall. Journal of Experimental Child Psychology, 47, 72-87.

Keeney, T. J., Canizzo, S. R., \& Flavell, J. H. (1967). Spontaneous and induced verbal rehearsal in a recall task. Child Development, 38, 953-966

KuCERA, H., \& Francis, W. N. (1967). Computational analysis of present-day English. Providence, RI: Brown University Press.

McCarTHY, D. (1970). McCarthy Scales of Children's Abilities. New York: The Psychological Corporation.

Raven, J. (1984). Raven's Coloured Progressive Matrices. London: H. K. Lewis \& Co.

Salamé, P., \& Baddeley, A. D. (1982). Disruption of short-term memory by unattended speech: Implications for the structure of working memory. Journal of Verbal Learning \& Verbal Behavior, 21, 150-164.

Stanovich, K. E., Cunningham, A. E., \& Ferman, D. J. (1984). Intelligence, cognitive skills, and early reading progress. Reading $\boldsymbol{R} \boldsymbol{e}-$ search Quarterly, 14, 278-303.

VyGoTsky, L. S. (1962). Thought and language. Cambridge, MA: MIT Press.

Walley, A. C., \& Metsala, J. L. (1992). Young children's age-ofacquisition estimates for spoken words. Memory \& Cognition, 20, 171-182.

\section{NOTE}

1. An effect size of .40 was adopted for the purposes of the power analyses of Experiments 1 and 2 because this corresponded to the correlation coefficient obtained between articulation rate and memory span for adults in Experiment 3.

(Manuscript received April 2, 1993; revision accepted for publication July 7,1993 .) 